\title{
Correction to: Brain and Human Body Modeling 2020
}

Sergey N. Makarov, Gregory M. Noetscher, and Aapo Nummenmaa

Correction to: S. N. Makarov et al. (eds.), Brain and Human Body Modeling 2020, https://doi.org/10.1007/978-3-030-45623-8

A chapter was removed from this Open Access volume and three subsequent chapters were moved into sections of the book which was not appropriate. The TOC has now been updated to match the correct order. 